\title{
Best Achievements in Pituitary and Adrenal Diseases in
} 2020

\author{
Chang Ho Ahn ${ }^{1,2}$, Jung Hee Kim ${ }^{1,3,4}$
}

${ }^{1}$ Department of Internal Medicine, Seoul National University College of Medicine, Seoul; ${ }^{2}$ Department of Internal Medicine, Seoul National University Bundang Hospital, Seongnam; ${ }^{3}$ Department of Internal Medicine, ${ }^{4}$ Pituitary Center, Seoul National University Hospital, Seoul, Korea

Significant progress in pituitary and adrenal diseases was made in 2020. This review presents major translational and clinical advances in research on pituitary and adrenal diseases, encompassing their epidemiology, pathogenesis, diagnosis, and clinical management. We address the controversy regarding the nomenclature of pituitary neuroendocrine tumors, omics-based molecular classification of pituitary adenomas, and novel drugs for Cushing's disease in the field of pituitary diseases. In the field of adrenal diseases, we cover big data-driven epidemiology of adrenal tumors, steroid profiling as a new diagnostic tool, and the utility of scoring systems in the decision-making process of managing primary aldosteronism. This brief article will broaden readers' understanding of pituitary and adrenal diseases.

Keywords: Pituitary neoplasms; Adrenal gland neoplasms; Steroids; Big data; Decision making

\section{INTRODUCTION}

In the past, we had only a rudimentary understanding of pituitary and adrenal disease due to the low disease prevalence and the small number of researchers in the field. However, advances in imaging technology and diagnostic tools have increased the prevalence of pituitary and adrenal disease, and research interest in this area has also grown over the past years. Moreover, with the advent of big data, omics analyses, and machine learning, the field of pituitary and adrenal diseases has evolved considerably in the last year.

The purpose of this review is to share new or evolving issues related to pituitary and adrenal diseases. Influential papers published between August 2019 and December 2020 are presented

Received: 27 January 2021, Revised: 31 January 2021,

Accepted: 5 February 2021

Corresponding author: Jung Hee Kim

Department of Internal Medicine, Seoul National University Hospital, Seoul National University College of Medicine, 101 Daehak-ro, Jongno-gu, Seoul 03080, Korea

Tel: +82-2-2072-4839, Fax: +82-2-764-2199, E-mail: jhkxingfu@gmail.com with a summary and a brief discussion of their clinical implications. Issues regarding pituitary diseases include nomenclature change, tumorigenesis, and new drugs. For adrenal diseases, epidemiology, steroid metabolomics, and the scoring system for primary aldosteronism are highlighted.

The advances in pituitary and adrenal diseases are summarized in Tables 1, 2.

\section{PITUITARY DISEASE}

\section{Nomenclature of pituitary adenoma: from pituitary adenoma to pituitary neuroendocrine tumors?}

In 2017, the International Pituitary Pathology Club (IPCC) proposed a revised nomenclature of adenohypophysial-cell tumors

Copyright () 2021 Korean Endocrine Society

This is an Open Access article distributed under the terms of the Creative Commons Attribution Non-Commercial License (https://creativecommons.org/ licenses/by-nc/4.0/) which permits unrestricted non-commercial use, distribution, and reproduction in any medium, provided the original work is properly cited. 
as pituitary neuroendocrine tumors (PitNETs) [1]. The IPCC claimed that the term "adenoma," which defines a benign tumor, does not appropriately describe the behavior of these tumors. Although a majority of pituitary tumors are indolent and non-invasive, a variable proportion may recur after complete resection and invade adjacent tissues such as the cavernous sinus. The term PitNET emphasizes that the biological spectrum of these tumors ranges from benign to malignant, and PitNETs should be considered as tumors with endocrine manifestations rather than simple endocrine diseases. However, this year, the Pituitary Society issued a position statement endorsing the term "pituitary adenoma." They explained that the nomenclature "PitNET" has alarming connotations and ignores the developmental origin of epithelial glandular tissues [2]. All neuroendocrine tumors are considered potentially malignant. In contrast, most pituitary tumors do not metastasize. Pituitary carcinomas are extremely rare $(0.2 \%$ of adenomectomies) [3]. The authors of the Pituitary Society argued that the term "PitNET" inappropriately labels most pituitary tumors with benign behavior as potentially malignant. Furthermore, they pointed out that neuroendocrine-specific markers, including synaptophysin and neuron-specific enolase, are also expressed in thyroid and adrenal tumors, suggesting that there is no clear distinction between the neuroendocrine gland and other endocrine glands. This question remains controversial, and the new term "PitNET" is not widely accepted yet.

\section{Molecular pathogenesis of pituitary tumors}

Neou et al. [4] reported a molecular classification of 134 PitNETs (or pituitary adenomas) using an integrated multi-omics approach. The PitNETs were mainly classified into the pituitary lineage, with five main histological subtypes-lactotroph, somatotroph, thyrotroph (POU1F1/PIT1 lineage), corticotroph (TBX19/TPIT lineage), and gonadotroph (NR5A1/SF1 lineage) - as well as the null-cell type. However, gonadotroph signatures were also found in some corticotroph and GNAS-wild somatotroph PitNETs, challenging the current definition of gonadotroph tumors. Moreover, in the TPIT lineage, corticotrophs were divided into three classes: the USP8-mutated with high hormonal activity, the USP8-wild-type with increased invasiveness, and large silent tumors with gonadotroph transdifferentiation. Genomic instability such as DNA hypomethylation and chromosomal instability was associated with POU1F1/PIT1 lineage differentiation. These findings improved our understanding of the pathophysiology of PitNETs.

Abboud et al. [5] reported the role of GPR101, an orphan G- protein coupled receptor, in the control of growth hormone (GH) secretion. Using a transgenic mouse and human pituitary tumor tissues, they showed that GPR101 activated Gs and Gq/11, which led to GH secretion, but not proliferation. This study provided new insights into the regulation of somatotroph function.

Another study reported a genetic basis of prolactinoma. A somatic hotspot mutation in splicing factor 3 subunit B1 (SF$B 1^{\mathrm{R} 625 \mathrm{H}}$ ) was discovered by whole-genome sequencing in 21 patients with prolactinoma and validated by digital polymerase chain reaction in 227 prolactinomas [6]. Patients with this mutation showed higher prolactin levels and shorter progression-free survival than those with non-mutated prolactinomas. Mechanistically, the $S F B 1^{\mathrm{R} 625 \mathrm{H}}$ mutation causes aberrant splicing of estrogen-related receptor gamma, which results in stronger binding of pituitary-specific positive transcription factor 1 .

\section{Novel drugs for Cushing's disease}

Although several drugs are available for Cushing's disease, there remains an unmet need for new and effective drugs because patients continue to suffer from the low tolerability and effectiveness of previous treatments. Phase 3 clinical trials for two new drugs for Cushing's syndrome were completed. Levoketoconazole is the $2 \mathrm{~S}, 4 \mathrm{R}$ enantiomer of ketoconazole. Ketoconazole, which has been used for Cushing's syndrome, is composed of two enantiomers, of which levoketoconazole is more potent for inhibiting enzymes of steroidogenesis. This allows a lower dose of levoketoconazole to achieve the same efficacy as the original ketoconazole. Levoketoconazole normalized urinary free cortisol levels after 6 months of a maintenance dose in approximately $30 \%$ of 94 patients enrolled in the phase 3 trial [7]. Adverse events led to study discontinuation in $13 \%$ of the patients. Liver enzymes were reversibly elevated to three times the upper normal limit in $11 \%$ of the patients.

Osilodrostat is a potent, oral, reversible inhibitor of $11 \beta$-hydroxylase. In a phase 3 trial, osilodrostat maintained a complete response in $53 \%$ of 137 patients without up-titration after week 12 [8]. Common side effects were nausea, headache, fatigue, and adrenal insufficiency. Hypertension and hypokalemia could occur due to the accumulation of mineralocorticoid precursors.

These two new drugs are orally administered and better tolerated than current medical treatments for Cushing's syndrome.

\section{ADRENAL DISEASE}

\section{Epidemiology of adrenal diseases}

The prevalence of adrenal tumors in previous reports was highly 


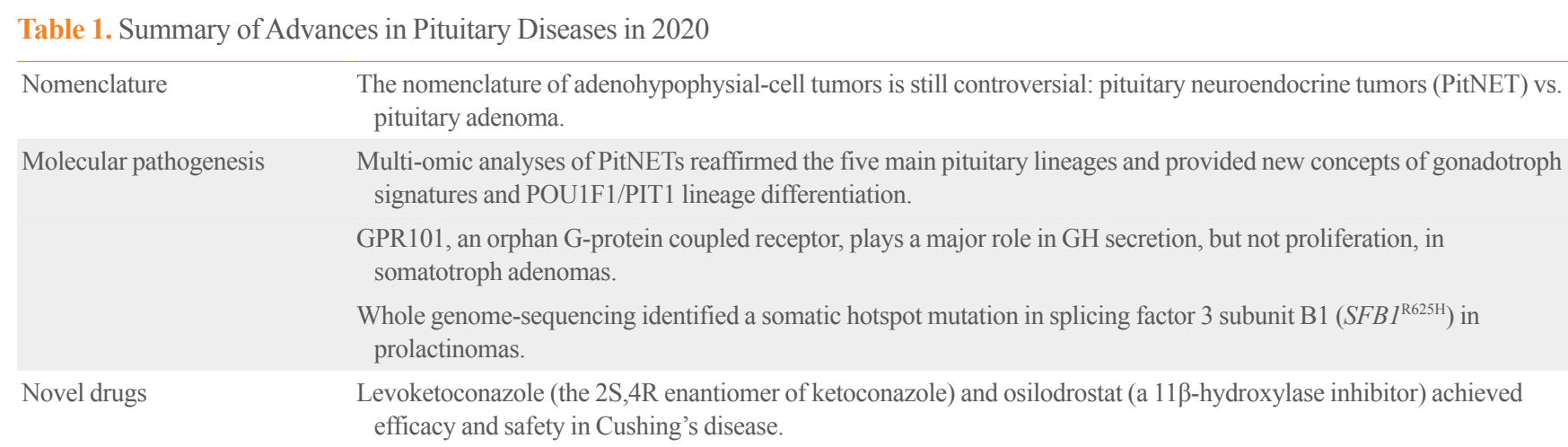

Table 2. Summary of Advances in Adrenal Diseases in 2020

\begin{tabular}{|c|c|}
\hline \multirow[t]{2}{*}{ Epidemiology } & $\begin{array}{l}\text { The prevalence of adrenal tumors was } 0.53 \% \text { and } 1.9 \% \text { in the overall population and subjects older than } 65 \text { years in a } \\
\text { community-based study. }\end{array}$ \\
\hline & $\begin{array}{l}\text { The prevalence of primary aldosteronism was } 11.3 \%, 15.7 \%, 21.6 \% \text {, and } 22.0 \% \text { in patients with normotension, stage } 1 \\
\text { hypertension, stage } 2 \text { hypertension, and resistant hypertension, respectively. }\end{array}$ \\
\hline \multirow[t]{2}{*}{ Steroid metabolomics } & $\begin{array}{l}\text { In Korean nationwide cohort studies, the prevalence of pheochromocytoma/paraganglioma and adrenal Cushing's } \\
\text { syndrome was } 2.13 \text { per } 100,000 \text { persons and } 2.34 \text { per } 100,000 \text { persons, respectively. }\end{array}$ \\
\hline & Plasma or urine steroid metabolomics discriminated adrenal cortical carcinoma from adrenal incidentalomas. \\
\hline \multirow[t]{2}{*}{$\begin{array}{l}\text { Scoring system for primary } \\
\text { aldosteronism }\end{array}$} & $\begin{array}{l}\text { Scoring systems for predicting unilateral primary aldosteronism may be useful for determining whether to perform } \\
\text { adrenalectomy when adrenal vein sampling is not available. }\end{array}$ \\
\hline & $\begin{array}{l}\text { Postoperative outcomes can be predicted through several preoperative factors, which were incorporated into the } \\
\text { Primary Aldosteronism Surgical Outcome (PASO) score. }\end{array}$ \\
\hline
\end{tabular}

variable, ranging from $1.0 \%$ to $8.7 \%$ [9]. A recent study reported the incidence and prevalence of adrenal tumors in a well-defined community database spanning more than 20 years [10]. In Olmsted County, MN, USA, the incidence of adrenal tumors increased drastically from 4.4 per 100,000 person-years in 1995 to 47.8 per 100,000 person-years in 2017 . The prevalence of adrenal tumors was $0.53 \%$ and $1.9 \%$ in the overall population and in patients older than 65 years, respectively. Among patients with adrenal tumors, $8.6 \%$ were diagnosed with malignancy (metastasis in $7.5 \%$ and adrenocortical carcinoma in $0.3 \%$ ). Another study investigated the prevalence of primary aldosteronism in a total of 1,014 patients whose blood pressure ranged from normotension to resistant hypertension [11]. All subjects completed an oral sodium suppression test to confirm the presence of primary aldosteronism. The prevalence of primary aldosteronism was $11.3 \%, 15.7 \%, 21.6 \%$, and $22.0 \%$ in patients with normotension, stage 1 hypertension, stage 2 hypertension, and resistant hypertension, respectively. This study suggested that primary aldosteronism is mainly unrecognized, and that the severity of aldosteronism is a continuum of renin-independent aldosterone production that parallels the severity of hypertension.
Epidemiological studies were also conducted with nationwide data from South Korea. The epidemiology of pheochromocytoma and paraganglioma (PPGL) was analyzed in the Korean National Health Insurance Service database. This nationwide study reported the age-standardized incidence of PPGL as 0.18 per 100,000 person-years and the prevalence of PPGL as 2.13 per 100,000 persons. Malignant PPGL accounted for $9.5 \%$ of all PPGLs. The 5-year survival rates for non-metastatic and metastatic PPGLs at diagnosis were $97 \%$ and $84 \%$, respectively. Another study reported the epidemiology of adrenal Cushing's syndrome in Korea [12]. This study also analyzed the National Health Insurance Service database and reported the age-standardized incidence rate of adrenal Cushing's syndrome as 0.13 per 100,000 person-years and the prevalence as 2.34 per 100,000 persons. The standardized mortality ratio was 3.0 for benign adrenal Cushing's syndrome and 13.1 for malignant adrenal Cushing's syndrome. Patients with adrenal Cushing's syndrome developed various complications (including hypertension, diabetes, cardiovascular disease, and osteoporosis) even after surgical treatment, underscoring the necessity of meticulous postoperative care. 


\section{Steroid metabolomics: new diagnostic and prognostic tools} for adrenal diseases

Steroid metabolomics involves detailed profiling of adrenal steroidogenesis. With the advent of liquid chromatography-mass spectrometry (LC-MS) technology, multiple steroids can be quantified in a single urine or serum sample. The usefulness of steroid metabolomics in diagnosing or subtyping adrenal gland diseases was investigated in recent studies. Bancos et al. [13] used urine steroid metabolomics in addition to the imaging characteristics of adrenal tumors for the differential diagnosis of adrenal incidentaloma. This EURINE-ACT study was a prospective, multinational, multicenter cohort study of patients with adrenal masses. Urinary steroid metabolites were quantified in urine samples using LC-MS. The imaging characteristics (unenhanced computed tomography [CT] attenuation $>20 \mathrm{HU}$ or positron emission tomography/CT or magnetic resonance imaging features), tumor diameter $>4 \mathrm{~cm}$, and the urinary steroid metabolomic profile were used to distinguish adrenocortical carcinoma from benign tumors. Each single criterion showed a high percentage of false-positive results, but the combination of the three criteria resulted in a high positive predictive value of $76.4 \%$. The use of urinary steroid metabolomics in addition to imaging studies can accurately rule out adrenocortical carcinoma among adrenal tumors. Another study provided novel insights into the bidirectional relationship between adrenal steroids and catecholamines using steroid metabolomics in plasma samples [14]. Plasma steroids were profiled using LC-MS in patients with PPGL and primary hypertension. Plasma levels of cortisol and its precursors and metabolites were significantly higher in patients with pheochromocytoma than in those with primary hypertension or paraganglioma. This study suggested that adrenal catecholamines can influence adrenal steroidogenesis.

\section{Scoring system for subtyping primary aldosteronism}

Primary aldosteronism is the most common cause of secondary hypertension. Attempts have been made to improve the subtyping of primary aldosteronism. The gold standard method for subtyping primary aldosteronism is adrenal vein sampling. However, adrenal vein sampling is an invasive test, and the rate of successful cannulation was around $70 \%$ even with an experienced interventional radiology team. A clinical prediction score was developed for subtyping primary aldosteronism without adrenal vein sampling [15]. Six parameters (higher aldosterone at screening and after confirmatory testing, lower potassium level, presence of nodules, higher nodule diameter, and absence of contralateral adrenal nodule) were indicative of unilateral pri- mary aldosteronism. The combination of these parameters resulted in an area under the receiver operating characteristic curve of 0.896 , with sensitivity and specificity of $91.7 \%$ and $79.3 \%$, respectively. This clinical score can be used to subtype primary aldosteronism when adrenal vein sampling is unavailable or fails. Another study suggested a clinical prediction score to subtype primary aldosteronism when adrenal vein sampling is only unilaterally successful [16]. The contralateral ratio (ratio of aldosterone/cortisol in the contralateral adrenal vein to that in the peripheral vein), in addition to aldosterone levels, potassium levels, and CT imaging, showed a high diagnostic value, with an area under the receiver operating characteristic curve of 0.971. These studies suggested new approaches to clinical decision-making when adrenal vein sampling is unavailable or insufficient.

\section{Surgical outcome score for unilateral primary aldosteronism}

In a Japanese nationwide cohort, called the Japan Primary Aldosteronism Study, 574 patients who were diagnosed with PA and underwent adrenalectomy were enrolled in the development of models that predicted postoperative blood pressure normalization at 6 months after surgery [17]. The researchers suggested the following independent predictors for postoperative outcome: duration of hypertension $\leq 7$ years, body mass index $\leq 25 \mathrm{~kg} / \mathrm{m}^{2}$, taking $\leq 1$ antihypertensive drug, absence of diabetes, and female sex. Their model presented an area under the receiver operating characteristic curve of 0.797 , showing moderate performance.

More recently, Williams et al. [18] and Burrello et al. [19] implemented the Primary Aldosteronism Surgical Outcome (PASO) score, a user-friendly online tool, in a subset of the PASO study group. They developed a prediction model for clinical outcomes after unilateral adrenalectomy for unilateral primary aldosteronism in a training dataset $(n=280)$ and validated it in a test dataset $(n=100)$. Intriguingly, they employed linear discriminant analysis, which maximizes the discrimination between groups by increasing precision with variable reduction. Six presurgical variables (duration of hypertension, sex, antihypertensive medication dosage, body mass index, target organ damage, and maximal size of the nodule) were integrated into a 25-point predictive score with an optimal cut-off of greater than 16 points, which showed an area under the curve of 0.839 . These results can be applied to the real practice in shared decision-making regarding adrenalectomy and the identification of patients who need regular postoperative care. 


\section{CONCLUSIONS}

In this review, we addressed pivotal advances in pituitary and adrenal diseases. We envision that these findings will improve patient care and inspire future research in the pituitary and adrenal field.

\section{CONFLICTS OF INTEREST}

No potential conflict of interest relevant to this article was reported.

\section{ORCID}

Chang Ho Ahn https://orcid.org/0000-0002-0702-0608

Jung Hee Kim https://orcid.org/0000-0003-1932-0234

\section{REFERENCES}

1. Asa SL, Casar-Borota O, Chanson P, Delgrange E, Earls P, Ezzat S, et al. From pituitary adenoma to pituitary neuroendocrine tumor (PitNET): an International Pituitary Pathology Club proposal. Endocr Relat Cancer 2017;24:C5-8.

2. Ho KK, Fleseriu M, Wass J, van der Lely A, Barkan A, Giustina A, et al. The tale in evolution: clarity, consistency and consultation, not contradiction and confusion. Pituitary 2020; 23:476-7.

3. Ho KK, Fleseriu M, Wass J, van der Lely A, Barkan A, Giustina A, et al. A tale of pituitary adenomas: to NET or not to NET: Pituitary Society position statement. Pituitary 2019; 22:569-73.

4. Neou M, Villa C, Armignacco R, Jouinot A, Raffin-Sanson ML, Septier A, et al. Pangenomic classification of pituitary neuroendocrine tumors. Cancer Cell 2020;37:123-34.

5. Abboud D, Daly AF, Dupuis N, Bahri MA, Inoue A, Chevigne A, et al. GPR101 drives growth hormone hypersecretion and gigantism in mice via constitutive activation of GS and $\mathrm{Gq} / 11$. Nat Commun 2020;11:4752.

6. Li C, Xie W, Rosenblum JS, Zhou J, Guo J, Miao Y, et al. Somatic SF3B1 hotspot mutation in prolactinomas. Nat Commun 2020;11:2506.

7. Fleseriu M, Pivonello R, Elenkova A, Salvatori R, Auchus RJ, Feelders RA, et al. Efficacy and safety of levoketoconazole in the treatment of endogenous Cushing's syndrome (SONICS): a phase 3, multicentre, open-label, single-arm trial. Lancet Diabetes Endocrinol 2019;7:855-65.
8. Pivonello R, Fleseriu M, Newell-Price J, Bertagna X, Findling J, Shimatsu A, et al. Efficacy and safety of osilodrostat in patients with Cushing's disease (LINC 3): a multicentre phase III study with a double-blind, randomised withdrawal phase. Lancet Diabetes Endocrinol 2020;8:748-61.

9. Lee JM, Kim MK, Ko SH, Koh JM, Kim BY, Kim SW, et al. Clinical guidelines for the management of adrenal incidentaloma. Endocrinol Metab (Seoul) 2017;32:200-18.

10. Ebbehoj A, Li D, Kaur RJ, Zhang C, Singh S, Li T, et al. Epidemiology of adrenal tumours in Olmsted County, Minnesota, USA: a population-based cohort study. Lancet Diabetes Endocrinol 2020;8:894-902.

11. Brown JM, Siddiqui M, Calhoun DA, Carey RM, Hopkins $\mathrm{PN}$, Williams GH, et al. The unrecognized prevalence of primary aldosteronism: a cross-sectional study. Ann Intern Med 2020;173:10-20.

12. Ahn CH, Kim JH, Park MY, Kim SW. Epidemiology and comorbidity of adrenal Cushing's syndrome: a nationwide cohort study. J Clin Endocrinol Metab 2020 Oct 19 [Epub]. https://doi.org/10.1210/clinem/dgaa752.

13. Bancos I, Taylor AE, Chortis V, Sitch AJ, Jenkinson C, Davidge-Pitts $\mathrm{CJ}$, et al. Urine steroid metabolomics for the differential diagnosis of adrenal incidentalomas in the EURINE-ACT study: a prospective test validation study. Lancet Diabetes Endocrinol 2020;8:773-81.

14. Constantinescu G, Langton K, Conrad C, Amar L, Assie G, Gimenez-Roqueplo AP, et al. Glucocorticoid excess in patients with pheochromocytoma compared with paraganglioma and other forms of hypertension. J Clin Endocrinol Metab 2020;105:e3374-83.

15. Burrello J, Burrello A, Pieroni J, Sconfienza E, Forestiero V, Rabbia $\mathrm{P}$, et al. Development and validation of prediction models for subtype diagnosis of patients with primary aldosteronism. J Clin Endocrinol Metab 2020;105:dgaa379.

16. Burrello J, Burrello A, Pieroni J, Sconfienza E, Forestiero V, Amongero M, et al. Prediction of hyperaldosteronism subtypes when adrenal vein sampling is unilaterally successful. Eur J Endocrinol 2020;183:657-67.

17. Morisaki M, Kurihara I, Itoh H, Naruse M, Takeda Y, Katabami T, et al. Predictors of clinical success after surgery for primary aldosteronism in the Japanese nationwide cohort. J Endocr Soc 2019;3:2012-22.

18. Williams TA, Lenders JW, Mulatero P, Burrello J, Rottenkolber M, Adolf C, et al. Outcomes after adrenalectomy for unilateral primary aldosteronism: an international consensus on outcome measures and analysis of remission rates in an 
international cohort. Lancet Diabetes Endocrinol 2017;5: 689-99.

19. Burrello J, Burrello A, Stowasser M, Nishikawa T, Quinkler M, Prejbisz A, et al. The primary aldosteronism surgical out- come score for the prediction of clinical outcomes after adrenalectomy for unilateral primary aldosteronism. Ann Surg 2020;272:1125-32. 